\title{
Iron deficiency and cognitive functions
}

This article was published in the following Dove Press journal:

Neuropsychiatric Disease and Treatment

10 November 2014

Number of times this article has been viewed

Ignacio Jáuregui-Lobera

Department of Nutrition and Bromatology, Pablo de Olavide University, Seville, Spain
Correspondence: Ignacio Jáuregui-Lobera Department of Nutrition and Bromatology, Pablo de Olavide University, Fernando IV,

24-26 Seville, Spain

Tel +34954280789

Fax +34954278 167

Email ignacio-ja@telefonica.net
Abstract: Micronutrient deficiencies, especially those related to iodine and iron, are linked to different cognitive impairments, as well as to potential long-term behavioral changes. Among the cognitive impairments caused by iron deficiency, those referring to attention span, intelligence, and sensory perception functions are mainly cited, as well as those associated with emotions and behavior, often directly related to the presence of iron deficiency anemia. In addition, iron deficiency without anemia may cause cognitive disturbances. At present, the prevalence of iron deficiency and iron deficiency anemia is $2 \%-6 \%$ among European children. Given the importance of iron deficiency relative to proper cognitive development and the alterations that can persist through adulthood as a result of this deficiency, the objective of this study was to review the current state of knowledge about this health problem. The relevance of iron deficiency and iron deficiency anemia, the distinction between the cognitive consequences of iron deficiency and those affecting specifically cognitive development, and the debate about the utility of iron supplements are the most relevant and controversial topics. Despite there being methodological differences among studies, there is some evidence that iron supplementation improves cognitive functions. Nevertheless, this must be confirmed by means of adequate follow-up studies among different groups.

Keywords: iron deficiency, anemia, cognitive functions, supplementation

\section{Introduction}

The importance of iron in cognitive functions is highlighted in some early studies. ${ }^{1,2}$ In those studies, some alterations with regard to psychomotor development and cognitive functions have been generally linked to the deficiencies of this mineral. Overall, micronutrient deficiencies are linked to different cognitive impairments, especially iodine and iron, ${ }^{3-6}$ as well as to potential long-term behavioral changes. ${ }^{6}$ Generally, a lack of iron in the neonatal period and early childhood is considered as a key to the development of disturbances in cognitive development. ${ }^{7-9}$ At the same time, there is little consensus about the effectiveness of possible preventive programs. ${ }^{10,11}$ Cognitive disturbances in general and visuomotor development have been related to iron deficiency (ID), ${ }^{12,13}$ and more recently a possible link between dieting, ID, and the possible presentation of cognitive alterations has been studied. ${ }^{14}$

Experimental studies show that ID is capable of causing cognitive impairment in animals and humans, ${ }^{15,16}$ with brain mitochondrial damage as a basis for these alterations. ${ }^{17}$ Among the cognitive impairments caused by ID, those related to attention span, intelligence, and sensory perception functions are mainly cited, as well as those related to emotions and behavior. Generally, these impairments have been related to iron deficiency anemia (IDA). In addition, it must be noted that ID without anemia may cause cognitive disturbances. ${ }^{11}$ With respect to ID and IDA, some concepts must be noted. Iron status is a continuum from IDA to ID without anemia to normal iron status with varying amounts of stored iron and finally to possible iron overload. ID is 
defined as a condition in which there are no mobilizable iron stores and in which signs of a compromised supply of iron to tissues, including the erythrocytes, are detected. When iron-deficient erythropoiesis occurs, hemoglobin concentrations are reduced to below optimal levels. The existence of anemia is considered when individual hemoglobin levels are below two standard deviations of the distribution mean for hemoglobin in an otherwise normal population of the same sex and age who are living at the same altitude. ${ }^{18}$

Twenty years ago, ID with anemia affected $>45 \%$ of children aged $<5$ years in developing countries and $>7 \%$ in developed countries. ${ }^{5}$ Currently, it is estimated to affect $2 \%-6 \%$ of European children, with a significant reduction in prevalence. Enriched foods and the use of supplements have contributed to that reduction, among other factors. ${ }^{19}$ Despite this improvement in the monitoring of ID, it seems that some health problems are associated with long-term deficits in cognitive and motor function and achievement gaps in education (small to gestational age infants, too short breastfeeding period, ID, severe and moderate protein energy malnutrition in early childhood, and intrauterine ID). In addition, behavioral differences have also been found in long-term follow-up studies of children who had protein energy malnutrition. ${ }^{6}$

In this field of study, the relevance of ID and IDA with respect to cognitive function is the main topic in the literature. The distinction between the cognitive consequences of ID and those affecting specifically cognitive development is another relevant point. The debate about the utility of iron supplements is also a topic that remains controversial.

Given the relevance of the problem of ID relative to proper cognitive development and the alterations that can persist through adulthood as a result of this deficiency, the objective of this study was to review the current state of knowledge about this health problem, trying to conclude with some practical conclusions.

\section{Material and methods}

\section{Searching process}

It was based on three relevant electronic databases (Medline, EMBASE, and Scopus), with a general strategy including terms related to "cognition" and "iron deficiency". The Medical Subjects Headings and the Boolean operators AND/OR were used for the searching process. The shared Mesh terms were: ((("Nutritional deficiencies"[Mesh]) OR ("Micronutrients"[Mesh]) OR ("Iron deficiency"[Mesh]) OR ("Iron deficiency anemia"[Mesh])) AND ((“Attention deficit”[Mesh]) OR (“'Intelligence”[Mesh]) OR ("Psychomotor
development"[Mesh]) OR ("Emotions"[Mesh]) OR (“Behavior"[Mesh]))).

Additional searching was carried out on references included in the papers, published reviews, and via hand searching. The literature search was mainly but not exclusively based on the last 15 years. Some former articles considered as pioneers and/ or especially relevant were also included.

Studies meeting the following criteria were included in the review: 1) studies focused on cognitive disturbances and ID and 2) controlled trials, randomized controlled trials, and comparative studies. The exclusion criteria were 1) case reports, 2) populations with nonspecific ID-related problems, 3) participants with special conditions/pathologies other than ID (eg, iodine deficiency), and 4) full text/abstract not available. Previous studies (reviews and meta-analysis) were considered as another source of articles, provided that they fitted the inclusion criteria. An analysis was carried out about references included in the papers as well as via hand searching.

The initial search yielded 269 references. References were included in an EndNote 9 library and screened on the basis of title and abstract. As a result, 26 duplicates were excluded. Thereafter, selected references were analyzed based on the full text. After applying the exclusion criteria, 89 studies were finally included.

\section{Thematic analysis}

To analyze the selected articles, a thematic analysis was used following the six-step framework of Braun and Clarke: ${ }^{20}$ becoming familiar with the data, creating initial codes, searching for themes, reviewing themes, defining and naming themes, and producing the report. Fragments of data that identify a significant feature of such data were acknowledged and grouped together into related themes. ${ }^{20,21}$ As a result, the following main different topics were obtained: anemia vs ID, neurocognitive development and educational performance, and the controversy about treatment and supplementation.

\section{Results}

\section{Anemia versus iron deficiency}

In a study about the effects of children's IDA on executive functions at 10 years, it has been found that those who had anemia showed slower reaction times, less accuracy, and higher latency peak N2 and smaller amplitud of the P300 wave (which is related to working memory) in the electroencephalogram (EEG). ${ }^{22,23}$

Compared with a control group (non-IDA infants), IDA infants (aged 3-15 months) showed lower scores in cognition, 
fine motor, and social/emotional areas. In addition, they had higher delta/theta and lower alpha power in EEG activity. ${ }^{24}$ Children aged 8-10 years were studied in order to determine the cognitive effects of IDA by means of the EEG P300 wave and some psychometric tests. ${ }^{25}$ As a result, P300 latency in the anemic girls was delayed as compared with the control group, but no statistically significant difference was observed for P300 latency and P300 amplitude between the control group and the anemic group. The psychometric test scores for intelligence quotient and transformed quotient were also better but not statistically significant in the control group of girls as compared with anemic girls. However, the hematocrit values showed a significant correlation, with the P300 wave latency showing that hematological status is associated with some effects on cognition. In a recent review, it was concluded that with respect to ID, of particular importance is the effect on the central nervous system, which leads to the defects in the cognition and learning processes in humans. ${ }^{26}$

A poorer object permanence and short-term memory encoding and/or retrieval have been shown in infants with IDA aged 9-12 months. These cognitive effects were attributable, in part, to IDA-related deficits in socioemotional function. Children with poor socioemotional performance seemed to be more vulnerable to the effects of IDA on cognitive function. ${ }^{27}$

If IDA occurs during the first 2 years of life, it is associated with poor psychomotor performance and changes in behavior, such as reduced levels of responsitivity to persons and stimuli, irritability, and inhibition. Different studies demonstrate that effects observed during infancy persist in the long-term. It has been reported that formerly anemic preschool children are less active, more inhibited, and more timid than the corresponding controls. ${ }^{28}$

ID and IDA may have some prolonged effects on behavior and development. ${ }^{29}$ In a review about the effects of ID in the first 2 years, it is concluded that the relationship between ID and IDA to cognitive and mental development in childhood is still unclear. Generally, follow-up studies have found poorer cognitive scores on measures of mental and cognitive functioning in the long run. ${ }^{30}$

Non-IDA predominantly due to anemia inflammation has been related to lower performance on tests of cognitive function, this likely being due to decreased delivery of iron to host tissues including the central nervous system. ${ }^{31}$ In fact, cognitive achievement is strongly related to hemoglobin level (higher hemoglobin levels result in better central nervous system function) and could be expected in anemic patients. $^{32}$
There is a debate about the cause of the cognitive impairment with respect to the anemia/ID. Is the impairment a result of ID per se or a combination of ID and anemia? How do these conditions interact? It has been found that cognitive function increases with increased hemoglobin concentration in children with ID but does not change with hemoglobin concentration in children with normal serum ferritin level. It seems that children with IDA consistently have the poorest cognitive function. Children with nonanemic ID but with high hemoglobin levels have significantly high cognitive function. So a dose-response relationship between hemoglobin and cognitive function in children with ID has been found, whereas no similar evidence is found in iron-sufficient children. ${ }^{33}$ Nevertheless, other authors support the idea that some cognitive symptoms get better with iron supplementation, this improvement being independent of the hemoglobin levels. ${ }^{34}$ In fact, a significant inverse relationship has been found between body iron and central executive function (planning time) in nonanemic college women. ${ }^{35}$

In a study focused on the effect of ID on cognitive development among children, the authors concluded that the effect of short-term iron treatment in anemic children aged $<2$ years is controversial. ${ }^{13}$ With respect to anemic children aged $>2$ years, it seems that iron treatment would be more effective. Finally, they stated that preventing IDA could produce benefits to development but that they would be small and transient.

\section{Neurocognitive development and educational performance}

A consequence of ID relies on alterations in cognition that occur among iron-deficient individuals during the early parts of their life cycle and perhaps at later times as well. While ID was presumed to cause most of its effects only if anemia was present, it is clear that many organs show morphologic, physiologic, and biochemical changes before there is any significant drop in hemoglobin concentration. ID is associated with alterations in many metabolic processes that may impact brain functioning (eg, mitochondria electron transport, neurotransmitter synthesis and degradation, protein synthesis, organogenesis). It is necessary to separate the developmental aspects of ID and neural functioning from the aspects of ID that could occur at any time in life. ${ }^{36}$

In a recent study, ${ }^{37}$ a chronic ID group showed significantly lower scores on language, environmental sound perception, and motor measures when compared with infants with normal nutritional iron status at 6 months and 14-18 months. The conclusion was that the development of 
language and motor skills and environmental sound perception appeared to be sensitive to the effects of chronic ID in infants. In fact, IDA produces alterations in cognitive processes related to visual attention and concept acquisition in children. ${ }^{38}$ A lower school achievement and poorer fine-hand movements have been described in children. ${ }^{39}$

For some authors, iron status does not play a major role in educational performance and intelligence of school-going adolescents, stating that several factors (apart from ID) affect educational performance and intelligence. ${ }^{40}$ Eden $^{41}$ highlights that children with ID and IDA, especially those in lower socioeconomic groups, are at high risk for neurodevelopmental impairment both from the ID itself as well as from central nervous system damage caused by the associated increased absorption of other metals like lead. With regards to the low socioeconomic groups, $26 \%$ of postinstitutionalized children have been shown to be iron deficient. ID is associated with neurobehavioral alterations months after postadoption, mediated by the effect on attention and activity levels (hyperactivity). ${ }^{42}$

Maternal genotypes at single nucleotide polymorphisms in the genes HFE (rs1799945) and (rs1800562), TF (rs3811647), and TMPRSS6 (rs1800562) are related to iron, hemoglobin, or transferrin levels. In a study by Lewis et $\mathrm{al}^{43}$ exploring the effect of prenatal iron levels on offspring cognition, the aforementioned mother's genotype loci were not associated with offspring intelligence quotient at age 8 years, so there is no evidence of an effect of exposure to low levels of iron (within the normal range) in pregnancy on offspring cognition at age 8 years. With respect to the genotypes, it has been shown that elevated iron levels may decrease cognitive speed in older individuals susceptible to cognitive impairment despite cognitive performance over 3 years not being associated with HFE C282Y genotype or iron parameters. ${ }^{44}$

There is evidence that, despite iron repletion, ID during the brain growth spurt alters metabolism and neurotransmission, myelination, and gene and protein profiles. ${ }^{45}$ In humans, there is compelling evidence that 6- to 24-month-old infants with IDA are at risk for poorer cognitive, motor, socioemotional, and neurophysiologic development in short- and long-term outcomes. Alterations in the mesolimbic pathway, where dopamine plays a major role in behavioral activation and inhibition, positive affect, and inherent reward may help explain altered socioemotional behavior in iron-deficient infants. ${ }^{46}$

Different functions and several ages seem to cause controversy in this field of study. On the one hand, in animals, deficits in motor activity are consistently associated with severe IDA, but adverse effects on performance in tests that target cognitive function have not been clearly shown. On the other hand, IDA resistant to iron administration (with the consequent failure of iron therapy) has been observed in most trials of children aged $<2$ years but not in older children. Similar observations have been made in rodents when IDA occurred before rather than after weaning. In children aged $>2$ years and in adolescents with IDA, evidence suggests cognitive or behavioral deficits. ${ }^{47}$ In a recent review ${ }^{48}$ it is stated that despite the clear elucidation of the neural mechanics of ID, there is not unequivocal support for a direct causal relationship between ID and abnormal development. Even accepting the evidence as being overwhelmingly in support of a direct causal link in experimental animals, we are far from being able to do so for humans, at least with any degree of specificity.

Apart from infants and children, women of reproductive age are specially affected by ID, and cognitive-behavioral consequences of that deficiency have been described in that population. ${ }^{49}$ Not only has ID (eg, as a result of a low intake of foods rich in bioavailable iron) been related to cognitive impairments but a high consumption of foods rich in inhibitors of iron (such as phytate, certain dietary fibres, and calcium) has too. ${ }^{16}$

\section{The controversy about treatment and supplementation}

Several benefits of iron supplementation, independent of increasing hemoglobin, such as those on immune function, physical performance, thermoregulation, cognition, and restless leg syndrome, among others, have been described. ${ }^{10}$ Considering the cognitive functions, the point is whether iron supplementation can improve them. ${ }^{50}$

With respect to the behavioral problems associated with ID, it seems that an early iron supplementation of marginally low birth weight infants does not affect cognitive functions at age 3.5 years but significantly reduces the prevalence of behavioral problems. ${ }^{51}$ For other authors, iron seems to have a modest effect on linear growth in deficient populations. ${ }^{52}$

Considering a positive effect of iron, does it depend on the previous ID (without anemia) or is it necessary to suffer from IDA? In this regard, Bruner et $\mathrm{al}^{53}$ reported the benefits of iron supplementation in an urban population of nonanemic irondeficient adolescent girls. That supplementation improved verbal learning and memory. Another study showed that in healthy formerly iron-deficient anemic children the altered cognitive function might be prolonged despite the iron 
treatment. The authors suggested that long-lasting changes in myelination and energy metabolism, perhaps especially in the hippocampus, might account for these long-term effects on an important aspect of human cognitive development. ${ }^{54}$ Devaki et al $^{55}$ tested two groups of adolescents (nonanemic ID and iron-deficient anemic) after 8 months of iron supplementation. This supplementation resulted in significant improvements in cognitive function and school achievements. In a shorter follow-up study (6 months of iron supplementation), Ebenezer et $\mathrm{al}^{56}$ did not find a significant impact on concentration levels and educational tests scores. Comparing iron-deficient anemic children with those who are iron replete, it seems that anemic children show faster motor development than the control children do. ${ }^{57}$ Another study highlights that there seems to be a dose-response relationship between hemoglobin and cognitive function in children with ID, whereas no similar evidence has been found in iron-sufficient children. ${ }^{58}$

Another relevant point is the moment to introduce the iron supplementation. In a study by Christian et $\mathrm{al}^{59}$ it was reported that fine motor functioning among offspring was positively associated with prenatal iron/folic acid supplementation in an area where ID was prevalent. In another study, Friel et $\mathrm{al}^{60}$ gave an iron supplementation to term breastfed infants (aged 1-6 months). With respect to the development effects, that supplementation resulted in higher visual acuity and developmental indexes at age 13 months. After controlling for background factors, children who had severe, chronic ID in infancy showed poorer arithmetic achievement and written expression, motor functioning, and some specific cognitive processes (spatial memory, selective recall, and tachistoscopic threshold), and this occurred 10 years after treatment. ${ }^{61}$ Sachdev et $\mathrm{al}^{62}$ have also reported that iron supplementation improves mental development modestly. This effect is particularly apparent for intelligence tests on children aged $>7$ years and in initially anemic or iron-deficient anemic subjects. There are controversial results with respect to the effect of iron therapy in children. While Sachdev et al state that there is no convincing evidence that iron treatment has an effect on mental development in children aged $<27$ months or on motor development, and Yalcin et $\mathrm{al}^{63}$ consider that short-term iron supplementation does not change developmental test scores despite the hematologic response in iron-sufficient healthy infants, others seem to report more positive results. Thus, Matiashvili et $\mathrm{al}^{64}$ found that children aged between 16 months and 24 months improved their performance in neurodevelopmental measures when they had received iron in a timely manner.
In preschool children, some improvements in cognitive functions have been reported after iron supplementation: eg, in discrimination, specifically selective attention. ${ }^{65}$ In a former study, it was reported that the administration of heme iron-fortified cookies might improve the intellectual performance of low-income preschool children. ${ }^{66}$ In addition, Soewondo et $\mathrm{al}^{38}$ and Idjradinata and Pollitt ${ }^{67}$ have reported cognitive improvements after iron supplementation in children. With respect to the cognitive development, Thompson et al ${ }^{68}$ state that iron supplementation produces a small improvement. It has been reported that iron treatment of young children with IDA has a positive short-term effect on psychomotor development or cognitive function, but the effect of longer-term treatment remains unclear. ${ }^{69}$ Not only iron but also a mixture of iron and folic acid supplementation seems to produce beneficial effects on cognition in young adolescent girls. ${ }^{70}$ On the contrary, Siegel et al ${ }^{71}$ state that neither combined nor individual supplements improve the performance on tasks such as A-not-B or fixation duration in infants. Solon et $\mathrm{al}^{72}$ reported similar results. These authors did not find any improvements in cognitive performance after supplementation with a multiple micronutrient-fortified drink. The effect of several drinks containing different micronutrients and sugar seems to be positive when those beverages are given alone. In other cases, the combination attenuates that positive effect on cognition. ${ }^{73}$ Baumgartner et $\mathrm{al}^{74}$ found that in children with poor iron and n-3 fatty acid status, iron supplementation improved verbal and nonverbal learning and memory, particularly in children with anemia. In contrast, docosahexaenoic acid/eicosapentaenoic acid supplementation had no benefits on cognition and impaired working memory in anemic children and long-term memory and retrieval in girls with ID.

Iron supplementation has been studied in adults with IDA by means of the Mini Mental State Examination, Wechsler Memory Scale - Revised, Wechsler Adult Intelligence Scale - Revised, event-related potentials, and EEG. As a result, authors concluded that IDA is a key in cognitive performance in adults, which can be partially reversed by treatment. ${ }^{75}$ In young women, Murray-Kolb and Beard ${ }^{76}$ have found that an improvement in serum ferritin after iron supplementation correlated to better cognitive performance, whereas a significant improvement in hemoglobin correlated to improved speed in completing the cognitive tasks. Recently, Leonard et $\mathrm{al}^{77}$ have analyzed some differences between iron-deficient and iron-sufficient young women with respect to their cognitive function after receiving placebo versus iron supplement. As a result, they have found out that 
change scores for impulsivity and attention were significantly greater in plasma ferritin improvers than in nonimprovers.

\section{Discussion}

Previous reviews on this field of study concluded that ID has a negative impact on cognition, behavior, and motor skills that can have long-term implications. ${ }^{13}$ Many studies highlight that the relationship between ID and cognitive consequences could be confounded by external factors such as low socioeconomic background. Apart from socioeconomic factors, ID seems to impact on areas such as the hippocampus, ${ }^{61}$ and the cognitive dysfunction might be due to mitochondrial damage. ${ }^{78}$ In addition, changes in brain dopamine metabolism are presumed to occur, ${ }^{1,2}$ as well as altered serotonergic neurotransmission ${ }^{5}$ and alterations in the dopamine receptors. ${ }^{79}$ In fact, brain ID and/or IDA result in major changes in dopamine levels in brain crucial areas. ${ }^{80}$ These changes related to central (brain) ID might cause different effects than those caused by IDA. More recently, Lozoff $^{46}$ has reported that alterations in the mesolimbic pathway, positive affect, and inherent reward might give support to explain the altered socioemotional behavior that has been described in children with ID. This author states that poorer motor sequencing and bimanual coordination and lower spontaneous eye blink rate in children with IDA are consistent with impaired function in the nigrostriatal pathway. Generally, nutrition has been shown to affect the brain's macrostructure (eg, development of brain areas such as the hippocampus), microstructure (eg, myelination of neurons), and level and operation of neurotransmitters (eg, dopamine levels or receptor numbers), all of which can have an impact on cognitive development. ${ }^{81}$ Specifically, nutrients such as iron and omega-3 - polyunsaturated fatty acids, in particular - might have specific effects on frontal lobe functioning. ${ }^{9}$

There are works in which it is possible to obtain a clear idea about the effects of ID and IDA on behavior and development. ${ }^{29}$ Generally, in non-IDA, there seems to be lower performance in cognitive functions, ${ }^{31}$ so a question arises as to whether ID, IDA, and anemia (other than IDA) may cause the same cognitive deficits. This question has an unclear response to date. Some studies correlate hemoglobin levels to cognitive function, ${ }^{33}$ while others report an improvement in cognitive functions after iron supplementation, which seems not to be correlated to those levels. ${ }^{34}$ Despite the specific effect of hemoglobin levels or iron status not being clear, IDA has proved to cause different cognitive alterations in all stages of life. A relevant effect of ID is caused during brain growth, as stated by Lozoff et al. ${ }^{45}$ For others, the effect of ID is not unequivocal considering a possible direct causal relationship between ID and abnormal development. ${ }^{48}$ Along with the work of Lozoff et al others seem to point out that there are critical periods in which ID might have long-term effects on cognition and behavior (eg, premature babies, infants aged $<1$ year, and preschool children). ${ }^{82-84}$ Apart from those critical periods, it seems that there are not any substantial different effects of ID or IDA on cognitive functions depending on the age reported clearly in the literature. With regards to the sex, it seems that there are no differences between boys and girls with ID in cognitive test performance. ${ }^{74}$

With more or fewer consequences and major or minor effects depending on age and sex, for example, there is a common practice that consists of supplementing with iron in case of ID and/or IDA. Here another debate arises. Is it useful?

There seem to be two tendencies considering the supplementation - on the one hand, the use of multimicronutrient supplements and, on the other hand, the use of specific micronutrients such as iron in this field of study. In addition, not always clear is the distinction between supplementation and fortification in the studies based on this topic. Nevertheless, this debate seems to be a perceptive problem. If both fortification and supplementation similarly involve the addition of micronutrients (vitamins or minerals) to the diet, why is there such a disparity in the perceptions of each? The fact is that while food fortification has been viewed by some as a nutritional triumph, individual supplementation with vitamins or minerals has, at best, been deemed unnecessary without underlying deficiency, and at worst been called potentially hazardous. So what are the differences between supplementation and fortification that make one more acceptable than the other ${ }^{85}$ So supplementation, fortification, or treatment should be considered without distinction in order to explore the cognitive improvements in case of ID and IDA.

Some authors report a behavioral improvement but not better results on cognitive function after iron supplementation, ${ }^{52}$ while others highlight an improvement in verbal learning/memory in ID (nonanemic) individuals. ${ }^{53}$ Despite years of treatment, there seem to be some cognitive alterations that persist. ${ }^{54}$ The controversial results focus not only on the long-term follow-up studies but on short-term ones too. For example, some authors state that an iron supplementation over a period of 8 months or 30 days is effective, while others point out that the supplementation during 6 months is not effective. $55,56,69$ 
Another relevant point highlights the moment to introduce the iron supplementation. When should it be introduced? There are a lot of studies indicating the success of an early iron supplementation: prenatal, ${ }^{59}$ age $1-6$ months,${ }^{60}$ age $<27$ months, ${ }^{62}$ age 16-24 months, ${ }^{64}$ and preschool, ${ }^{65,66}$ among others.

Finally, there are controversial results with respect to the "mixtures" used as supplements. Thus, a beneficial effect of iron and folic acid supplementation has been reported..$^{70} \mathrm{On}$ the contrary, Siegel et $\mathrm{al}^{71}$ and Solon et $\mathrm{al}^{72}$ do not find positive results with combined supplements. For others, the combination of supplements might attenuate some positive effects. ${ }^{73}$ With respect to the specific action of polyunsaturated fatty acids, Baumgartner et a ${ }^{74}$ found that docosahexaenoic acid/ eicosapentaenoic acid supplementation had no benefits on cognition and impaired working memory in anemic children and long-term memory and retrieval in girls with ID.

Despite iron excess cannot be excreted by the human body (in contrast with other nutrients), iron supplementation is often recommended, that supplementation not always being necessary. Iron supplements seem to be beneficial in iron-deficient children, but there is a risk of adverse effects in those who are iron replete. It has been suggested that excessive iron supplementation of infants may have adverse effects on growth, risk of infections, and even on cognitive development. In addition, excessive iron intake can have negative effects on brain development. ${ }^{86}$ So in populations with a low prevalence of ID, general supplementation should be avoided. The same applies to iron-fortified foods. In this regard, more studies are needed to better determine the risks and benefits of iron supplementation and iron-fortified foods given to iron-deficient and iron-sufficient children. ${ }^{86}$

Despite there being methodological differences among studies, there is some evidence that iron supplementation may improve some functions, such as attention, concentration, and intelligence. Nevertheless, these results require confirmation with well-powered, blinded, independently funded, randomized controlled trials of at least 1 year's duration in different age groups including children, adolescents, adults, and older people and across all levels of baseline iron status. ${ }^{87,88}$ Many studies differ in the characteristics of the study population, definition of exposure, type of treatment, and confounders, so it is difficult to assess a causal relationship between ID and IDA and cognitive and mental development in childhood, ${ }^{30}$ and the same applies to the use of supplements.

Maybe, an early-introduced correct (adequate) diet is better than the use of supplements. Thus, some studies suggest that increased meat intake by breastfed infants aged $>6$ months would adequately support iron requirements. ${ }^{89}$

\section{Conclusion}

There is a consensus on the fact that ID has a negative impact on cognition, behavior, and motor skills. With respect to this negative impact, its causal link with ID might be confounded by external variables such as socioeconomic status.

ID, IDA, and non-ID anemia may cause some cognitive deficits, but it remains unclear if those deficits are the same. These cognitive deficits may appear at any age. Levels of hemoglobin seem to correlate to cognitive performance, but iron supplementation improves cognitive functions regardless of the hemoglobin levels.

Despite the possible action of confounders, ID is clearly related to alterations in areas such as the hippocampus, mitochondrial damage, brain dopamine metabolism, and myelination.

The use of supplements (fortification, treatment) must be based on well-established indications, avoiding their use as a routine. The use of multisupplements does not seem to add a plus with respect to the use of specific supplements. The success of the supplementation might be based on an early prescription after having diagnosed an iron deficiency. It remains controversial whether that supplementation is successful or not, depending on the timing of the therapy (eg, critical periods).

More studies of an appropriate duration in different age groups (children, adolescents, adults, and older people) and across all levels of baseline iron status are required.

\section{Disclosure}

The author reports no conflicts of interest in this work.

\section{References}

1. Beard J. One person's view of iron deficiency, development, and cognitive function. Am J Clin Nutr. 1995;62:709-710.

2. Kretchmer N, Beard JL, Carlson S. The role of nutrition in the development of normal cognition. Am J Clin Nutr. 1996;63:997S-1001S.

3. Fanjiang G, Kleinman RE. Nutrition and performance in children. Curr Opin Clin Nutr. 2007;10:342-347.

4. Black RE. Global distribution and disease burden related to micronutrient deficiencies. Nestle Nutr Inst Workshop Ser. 2014;78:21-28.

5. Grantham-McGregor SM, Ani CC. The role of micronutrients in psychomotor and cognitive development. Br Med Bull. 1999;55:511-527.

6. Grantham-McGregor SM, Walker SP, Chang S. Nutritional deficiencies and later behavioural development. Proc Nutr Soc. 2000;59:47-54.

7. Bodnar LM, Cogswell ME, McDonald T. Have we forgotten the significance of postpartum iron deficiency? Am J Obstet Gynecol. 2005;193:36-44.

8. Benton D, ILSI Europe a.i.s.b.1. Micronutrient status, cognition and behavioral problems in childhood. Eur J Nutr. 2008;47 Suppl 3:38-50.

9. Bryan J, Osendarp S, Hughes D, et al. Nutrients for cognitive development in school-aged children. Nutr Rev. 2004;62:295-306.

10. Agarwal R. Non-hematological benefits of iron. Am J Nephrol. 2007; 27:565-571.

11. Black MM. Micronutrient deficiencies and cognitive functioning. J Nutr. 2003;133:3927S-3931S. 
12. Gonzalez HF, Malpeli A, Etchegoyen G, et al. Acquisition of visuomotor abilities and intellectual quotient in children aged 4-10 years: relationship with micronutrient nutritional status. Biol Trace Elem Res. 2007;120:92-101.

13. Grantham-McGregor S, Ani C. A review of studies on the effect of iron deficiency on cognitive development in children. J Nutr. 2001;131:649S-668S

14. Green MW, Elliman NA. Are dieting-related cognitive impairments a function of iron status? Br J Nutr. 2013;109:184-192.

15. Tamura T, Goldenberg RL, Hou J, et al. Cord serum ferritin concentrations and mental and psychomotor development of children at five years of age. J Pediatr. 2002;140:165-170.

16. Sandstead HH, Frederickson CJ, Penland JG. History of zinc as related to brain function. J Nutr. 2000;130:496S-502S.

17. Atamna H, Walter PB, Ames BN. The role of heme and iron-sulfur clusters in mitochondrial biogenesis, maintenance, and decay with age. Arch Biochem Biophys. 2002;397:345-353.

18. World Health Organization. Iron Deficiency Anaemia. Assessment, Prevention, and Control. A Guide for Programme Managers. Geneva, Switzerland: World Health Organization; 2001.

19. Sánchez Brevers A. Monografía Sobre la Anemia Ferropénica [dissertation]. University of Cantabria (Spain); 2013.

20. Braun V, Clarke V. Using thematic analysis in psychology. Qual Res Psychol. 2006;3:77-101.

21. Aveyard H. Doing a Literature Review in Health and Social Care. 2nd ed. Berkshire, UK: McGraw-Hill; 2010.

22. Alloway TP. What do we know about the long-term cognitive effects of iron-deficiency anemia in infancy? Dev Med Child Neurol. 2013;55:401-402.

23. Algarin C, Nelson CA, Peirano P, et al. Iron-deficiency anemia in infancy and poorer cognitive inhibitory control at age 10 years. Dev Med Child Neurol. 2013;55:453-458.

24. Ayala R, Otero GA, Porcayo Mercado R, et al. Delayed CNS maturation in iron-deficient anaemic infants. Nutr Neurosci. 2008;11:61-68.

25. Bandhu R, Shankar N, Tandon OP, et al. Event related potentials in anemic school-going girls of age group 8 to 10 years. Indian J Physiol Pharmacol. 2011;55:234-240.

26. Batra J, Sood A. Iron deficiency anaemia: effect on cognitive development in children: a review. Indian J Clin Biochem. 2005;20:119-125.

27. Carter RC, Jacobson JL, Burden MJ, et al. Iron deficiency anemia and cognitive function in infancy. Pediatrics. 2010;126:e427-e434.

28. De Andraca Oyarzun I, Salas Aliaga I, de la Parra Cieciwa A, et al. Motherchild interaction and child behavior in preschool children with a history of iron-deficiency anemia in infancy. Arch Lat Nutr. 1993;43:191-198.

29. Deinard AS, List A, Lindgren B, et al. Cognitive deficits in iron-deficient and iron-deficient anemic children. J Pediatr. 1986;108:681-689.

30. Glazer Y, Bilenko N. Effect of iron deficiency and iron deficiency anemia in the first two years of life on cognitive and mental development during childhood. Harefuah. 2010;149:309-314,335.

31. Olson CL, Acosta LP, Hochberg NS, et al. Anemia of inflammation is related to cognitive impairment among children in Leyte, the Philippines. PLoS Neglect Trop D. 2009;3:e533.

32. Petranovic D, Batinac T, Petranovic D, et al. Iron deficiency anaemia influences cognitive functions. Med Hypotheses. 2008;70:70-72.

33. Sungthong R, Mo-suwan L, Chongsuvivatwong V. Effects of haemoglobin and serum ferritin on cognitive function in school children. Asia Pac J Clin Nutr. 2002;11:117-122.

34. Agaoglu L, Torun O, Unuvar E, et al. Effects of iron deficiency anemia on cognitive function in children. Arzneimittel-Forschung. 2007;57:426-430.

35. Blanton CA, Green MW, Kretsch MJ. Body iron is associated with cognitive executive planning function in college women. Br J Nutr. 2013;109:906-913.

36. Beard JL. Iron deficiency and neural development: an update. Arch Lat Nutr. 1999;49:34S-39S

37. Beltran-Navarro B, Matute E, Vasquez-Garibay E, et al. Effect of chronic iron deficiency on neuropsychological domains in infants. J Child Neurol. 2012;27:297-303.
38. Soewondo S, Husaini M, Pollitt E. Effects of iron deficiency on attention and learning processes in preschool children: Bandung, Indonesia. Am J Clin Nutr. 1989;50:667-674.

39. Walter T. Effect of iron-deficiency anemia on cognitive skills and neuromaturation in infancy and childhood. Food Nutr Bull. 2003; 24:S104-S110.

40. Dissanayake DS, Kumarasiri PVR, Nugegoda DB, et al. The association of iron status with educational performance and intelligence among adolescents. Ceylon Med J. 2009;54:75-79.

41. Eden AN. Iron deficiency and impaired cognition in toddlers: an underestimated and undertreated problem. Paediatr Drugs. 2005;7:347-352.

42. Fuglestad AJ, Georgieff MK, Iverson SL, et al. Iron deficiency after arrival is associated with general cognitive and behavioral impairment in post-institutionalized children adopted from Eastern Europe. Matern Child Health J. 2013;17:1080-1087.

43. Lewis SJ, Bonilla C, Brion MJ, et al. Maternal iron levels early in pregnancy are not associated with offspring IQ score at age 8, findings from a mendelian randomization study. Eur J Clin Nutr. 2014;68:496-502.

44. Schiepers OJG, van Boxtel MPJ, de Groot RHM, et al. Serum iron parameters, hfe c282y genotype, and cognitive performance in older adults: results from the FACIT study. J Gerontol A Biol Sci Med Sci. 2010;65:1312-1321.

45. Lozoff B, Armony-Sivan R, Kaciroti N, et al. Eyeblinking rates are slower in infants with iron-deficiency anemia than in nonanemic irondeficient or iron-sufficient infants. J Nutr. 2010;140:1057-1061.

46. Lozoff B. Early iron deficiency has brain and behavior effects consistent with dopaminergic dysfunction. J Nutr. 2011;141:740S-746S.

47. McCann JC, Ames BN. An overview of evidence for a causal relation between iron deficiency during development and deficits in cognitive or behavioral function. Am J Clin Nutr. 2007;85:931-945.

48. Thomas DG, Grant SL, Aubuchon-Endsley NL. The role of iron in neurocognitive development. Dev Neuropsychol. 2009;34:196-222.

49. Murray-Kolb LE. Iron status and neuropsychological consequences in women of reproductive age: what do we know and where are we headed? J Nutr. 2011;141:747S-755S.

50. Ashby D. Can iron supplementation improve cognitive functioning? Lancet. 1996;348:973.

51. Berglund SK, Westrup B, Hagglof B, et al. Effects of iron supplementation of lbw infants on cognition and behavior at 3 years. Pediatrics. 2013;131:47-55.

52. Bhandari N, Bahl R, Taneja S. Effect of micronutrient supplementation on linear growth of children. Br J Nutr. 2001;85:S131-S137.

53. Bruner AB, Joffe A, Duggan AK, et al. Randomised study of cognitive effects of iron supplementation in non-anaemic iron-deficient adolescent girls. Lancet. 1996;348:992-996.

54. Congdon EL, Westerlund A, Algarin CR, et al. Iron deficiency in infancy is associated with altered neural correlates of recognition memory at 10 years. J Pediatr. 2012;160:1027-1033.

55. Devaki PB, Chandra RK, Geisser P. Effects of oral iron(iii) hydroxide polymaltose complex supplementation on hemoglobin increase, cognitive function, affective behavior and scholastic performance of adolescents with varying iron status: a single centre prospective placebo controlled study. Arzneimittel-Forschung. 2009;59:303-310.

56. Ebenezer R, Gunawardena K, Kumarendran B, et al. Cluster-randomised trial of the impact of school-based deworming and iron supplementation on the cognitive abilities of schoolchildren in Sri Lanka's plantation sector. Trop Med Int Health. 2013;18:942-951.

57. Harahap H, Jahari AB, Husaini MA, et al. Effects of an energy and micronutrient supplement on iron deficiency anemia, physical activity and motor and mental development in undernourished children in Indonesia. Eur J Clin Nutr. 2000;54:S114-S119.

58. Sungthong R, Mo-suwan L, Chongsuvivatwong V, et al. Onceweekly and 5-days a week iron supplementation differentially affect cognitive function but not school performance in Thai children. J Nutr. 2004; $134: 2349-2354$.

59. Christian P, Murray-Kolb LE, Khatry SK, et al. Prenatal micronutrient supplementation and intellectual and motor function in early schoolaged children in Nepal. JAMA. 2010;304:2716-2723. 
60. Friel JK, Aziz K, Andrews WL, et al. A doublemasked, randomized control trial of iron supplementation in early infancy in healthy term breast-fed infants. J Pediatr. 2003;143:582-586.

61. Lozoff B, Jimenez E, Hagen J, et al. Poorer behavioral and developmental outcome more than 10 years after treatment for iron deficiency in infancy. Pediatrics. 2000;105:E51.

62. Sachdev H, Gera T, Nestel P. Effect of iron supplementation on mental and motor development in children: systematic review of randomised controlled trials. Public Health Nutr. 2005;8:117-132.

63. Yalcin SS, Yurdakok K, Acikgoz D, et al. Short-term developmental outcome of iron prophylaxis in infants. Pediatr Int. 2000;42:625-630.

64. Matiashvili K, Manjavidze N, Ghonghadze T. Influence of ferrotherapy on psychomotor development of children of yearly age with iron deficiency anemia. Georgian Med News. 2012;206:38-41.

65. Metallinos-Katsaras E, Valassi-Adam E, Dewey KG, et al. Effect of iron supplementation on cognition in Greek preschoolers. Eur J Clin Nutr. 2004;58:1532-1542.

66. Salinas-Pielago JE, Vega-Dienstmaier JM, Rojas-Oblitas M. Effect of biscuits fortified with haem iron on the intellectual status of pre-school children. Rev Neurol. 1998;27:400-404.

67. Idjradinata P, Pollitt E. Reversal of developmental delays in iron-deficient anaemic infants treated with iron. Lancet. 1993;341(8836):1-4.

68. Thompson J, Biggs BA, Pasricha SR. Effects of daily iron supplementation in 2- to 5-year-old children: systematic review and meta-analysis. Pediatrics. 2013;131:739-753.

69. Wang B, Zhan S, Gong T, et al. Iron therapy for improving psychomotor development and cognitive function in children under the age of three with iron deficiency anaemia. Cochrane Database Syst Rev. 2013;6:CD001444.

70. Sen A, Kanani SJ. Impact of iron-folic acid supplementation on cognitive abilities of school girls in Vadodara. Indian Pediatrics. 2009;46:137-143.

71. Siegel EH, Kordas K, Stoltzfus RJ, et al. Inconsistent effects of ironfolic acid and/or zinc supplementation on the cognitive development of infants. J Health Popul Nutr. 2011;29:593-604.

72. Solon FS, Sarol JN Jr, Bernardo ABI, et al. Effect of a multiplemicronutrient-fortified fruit powder beverage on the nutrition status, physical fitness, and cognitive performance of schoolchildren in the Philippines. Food Nutr Bull. 2003;24:S129-S140.

73. Taljaard C, Covic NM, van Graan AE, et al. Effects of a multimicronutrient-fortified beverage, with and without sugar, on growth and cognition in South African schoolchildren: a randomised, double-blind, controlled intervention. Br J Nutr. 2013;110:2271-2284.

74. Baumgartner J, Smuts CM, Malan L, et al. Effects of iron and n-3 fatty acid supplementation, alone and in combination, on cognition in school children: a randomized, double-blind, placebo-controlled intervention in South Africa. Am J Clin Nutr. 2012;96:1327-1338.
75. Khedr E, Hamed SA, Elbeih E, et al. Iron states and cognitive abilities in young adults: neuropsychological and neurophysiological assessment. Eur Arch Psychiatry Clin Neurosci. 2008;258: 489-496.

76. Murray-Kolb LE, Beard JL. Iron treatment normalizes cognitive functioning in young women. Am J Clin Nutr. 2007;85:778-787.

77. Leonard AJ, Chalmers KA, Collins CE, et al. A study of the effects of latent iron deficiency on measures of cognition: a pilot randomised controlled trial of iron supplementation in young women. Nutrients. 2014;6:2419-2435.

78. Ames BN. The metabolic tune-up: metabolic harmony and disease prevention. J Nutr. 2003;133:1544S-1548S.

79. Pollitt E. The developmental and probabilistic nature of the functional consequences of iron-deficiency anemia in children. J Nutr. 2001;131:669S-675S

80. Youdim MB, Yehuda S. The neurochemical basis of cognitive deficits induced by brain iron deficiency: involvement of dopamine-opiate system. Cell Mol Biol. 2000;46:491-500.

81. Lozoff B, Beard J, Connor J, et al. Long-lasting neural and behavioral effects of iron deficiency in infancy. Nutr Rev. 2006;64:S34-S43, discussion S72-S91.

82. Armony-Sivan R, Kaplan-Estrin M, Jacobson SW, et al. Iron-deficiency anemia in infancy and mother-infant interaction during feeding. $J$ Dev Behav Pediatr. 2010;31:326-332.

83. Lozoff B, Castillo M, Clark KM, et al. Iron supplementation in infancy contributes to more adaptive behaviour at 10 years of age. J Nutr. 2014;144:838-845.

84. Yehuda S, Yehuda M. Long lasting effects of infancy iron deficiencypreliminary results. J Neurol Trans Suppl. 2006;71:197-200.

85. Connolly KM. Supplementation versus food fortification. The unfounded disparity in perception. Total Health. 2011. Available from: http://www.totalhealthmagazine.com/articles/diet/supplementationversus-food-fortification-the-unfounded-disparity-in-perception.html. Accessed September 22, 2014.

86. Domellof M. Benefits and harms of iron supplementation in irondeficient and iron-sufficient children. Nestle Nutr Workshop Ser Pediatr Program. 2010;65:153-165.

87. Logan S, Martins S, Gilbert R. Iron therapy for improving psychomotor development and cognitive function in children under the age of three with iron deficiency anaemia. Cochrane Database Syst Rev. 2001;(2):CD001444

88. Falkingham M, Abdelhamid A, Curtis P, et al. The effects of oral iron supplementation on cognition in older children and adults: a systematic review and meta-analysis. Nutr J. 2010;9:4

89. Krebs NF. Dietary zinc and iron sources, physical growth and cognitive development of breastfed infants. J Nutr. 2000;130:358S-360S.
Neuropsychiatric Disease and Treatment

\section{Publish your work in this journal}

Neuropsychiatric Disease and Treatment is an international, peerreviewed journal of clinical therapeutics and pharmacology focusing on concise rapid reporting of clinical or pre-clinical studies on a range of neuropsychiatric and neurological disorders. This journa is indexed on PubMed Central, the 'PsycINFO' database and CAS,

\section{Dovepress}

and is the official journal of The International Neuropsychiatric Association (INA). The manuscript management system is completely online and includes a very quick and fair peer-review system, which is all easy to use. Visit http://www.dovepress.com/testimonials.php to read real quotes from published authors. 